\title{
The use of alternatives to clopidogrel in flow-diversion treatment with the Pipeline embolization device
}

\author{
Elias Atallah, MD, Hassan Saad, MD, Kimon Bekelis, MD, Nohra Chalouhi, MD, \\ Stavropoula Tjoumakaris, MD, David Hasan, MD, Jorge Eller, MD, David Stidd, MD, \\ Robert H. Rosenwasser, MD, and Pascal Jabbour, MD \\ Department of Neurological Surgery, Thomas Jefferson University and Jefferson Hospital for Neuroscience, Philadelphia, \\ Pennsylvania
}

OBJECTIVE Thromboembolic complications continue to be encountered with Pipeline embolization devices (PEDs) despite routine clopidogrel/aspirin antiplatelet therapy. This study examined the safety and efficacy of prasugrel in the management of clopidogrel-resistant patients treated for cerebral aneurysms.

METHODS Four hundred thirty-seven consecutive patients were identified between January 2011 and May 2016. Patients allergic, or having less than $30 \%$ platelet inhibition, to a daily 75 -mg dose of clopidogrel received $10 \mathrm{mg}$ of prasugrel daily $(n=20)$ or $90 \mathrm{mg}$ of ticagrelor twice daily $(n=2)$. The mean $( \pm S D)$ follow-up duration was $15.8 \pm 12.4$ months. The primary outcome was the modified Rankin Scale (mRS) score registered before discharge and at each follow-up visit. To control confounding, multivariable mixed-effects logistic regression and propensity score conditioning were used.

RESULTS Twenty-six (5.9\%) of 437 patients presented with a subarachnoid hemorrhage (SAH). The mean patient age was 56.3 years, and 62 were women (14.2\%). One of the 7 patients lost to follow-up received prasugrel. One patient was allergic to clopidogrel and prasugrel simultaneously. All patients receiving prasugrel or ticagrelor $(n=22)$ had an $m R S$ score $\leq 2$ on their latest follow-up visit (mean score $0.67 \pm 1.15$ ). In a multivariate analysis, clopidogrel did not affect the $\mathrm{mRS}$ score on last follow-up $(p=0.14)$. Multivariable logistic regression showed that clopidogrel was not associated with an increased long-term recurrence rate ( $\mathrm{OR} 0.17,95 \% \mathrm{Cl} 0.01-2.70, p=0.21)$, an increased thromboembolic complication rate (OR $0.46,95 \% \mathrm{Cl} 0.12-1.67, \mathrm{p}=0.24)$, or an increased hemorrhagic event rate (OR $0.39,95 \% \mathrm{Cl} 0.91-1.64, \mathrm{p}$ $=0.20$ ). None of the patients receiving prasugrel or ticagrelor died or suffered a long-term recurrence or a hemorrhagic event; only 1 patient suffered from mild aphasia subsequent to a thromboembolic event. Three patients taking clopidogrel died during the study: 2 from acute SAH and 1 from intraparenchymal hemorrhage. Clopidogrel was not associated with an increased mortality rate $(\mathrm{OR} 2.18,95 \% \mathrm{Cl} 0.11-43.27, \mathrm{p}=0.61)$. The same associations were present in propensity score-adjusted models.

CONCLUSIONS In a cohort of patients treated with PEDs, prasugrel (10 mg/day) was a safe alternative to clopidogrelresistant or clopidogrel-allergic patients, or nonresponders.

https://thejns.org/doi/abs/10.3171/2017.5.JNS162663

KEY WORDS aneurysm; Pipeline embolization device; prasugrel; ticagrelor; clopidogrel; alternatives; vascular disorders

$\mathrm{T}$ HE superiority of coil embolization as compared with clip placement in a certain category of brain aneurysms has been demonstrated by the International Subarachnoid Aneurysm Trial. ${ }^{10}$ Since its 2011 FDA approval, the Pipeline embolization device (PED;
Covidien) has been a favored option in treating cerebral aneurysms. ${ }^{16}$ The PED is a self-expanding stent with $30 \%-35 \%$ metal surface area coverage that diverts blood flow from the aneurysm lumen to the downstream arteries, causing aneurysm sac thrombosis. ${ }^{4}$ However, there is

ABBREVIATIONS DAPT = dual antiplatelet therapy; DSA = digital subtraction angiography; $m$ RS = modified Rankin Scale; $\mathrm{PED}=\mathrm{Pipeline}$ embolization device; $\mathrm{SAH}=$ subarachnoid hemorrhage; TRITON-TIMI 38 = Trial to Assess Improvement in Therapeutic Outcomes by Optimizing Platelet Inhibition with Prasugrel-Thrombolysis In Myocardial Infarction 38.

SUBMITTED October 20, 2016. ACCEPTED May 30, 2017

INCLUDE WHEN CITING Published online December 8, 2017; DOI: 10.3171/2017.5.JNS162663. 
a window of time until full luminal endothelialization of the PED occurs, and during this time the patient is at a high risk of thromboembolic events. ${ }^{12}$ Despite the encouraging preliminary outcomes, there have been multiple descriptions of immediate or delayed thrombotic or hemorrhagic complications following PED deployment. ${ }^{2,14}$ The use of dual antiplatelet therapy (DAPT) with aspirin and clopidogrel has been recommended for preventing these complications. ${ }^{13}$ However, Delgado Almandoz et al. demonstrated that thromboembolic complications continue to be encountered, particularly with PEDs, despite routine DAPT. Approximately $30 \%$ of patients exhibit antiplatelet resistance. ${ }^{1}$ This variability in the response to clopidogrel is implied by the degree of insufficient platelet inhibition in CYP2C19 heterozygous patients. A small number of centers have substituted clopidogrel with different antiaggregation drugs, such as prasugrel or ticagrelor, in the management of these resistant cases. ${ }^{6,15}$ Prasugrel and ticagrelor achieve more potent and rapid inhibition of platelet aggregation and decreased intersubject response variability. ${ }^{7,9}$ In our study, we identified all patients who were resistant to clopidogrel. These patients were dispensed prasugrel or ticagrelor to achieve optimal platelet inhibition, which allowed them to participate in flow-diverting stent treatment. Our study demonstrates the safety and efficacy of prasugrel and ticagrelor as alternative antiplatelet agents while dispensed in conjunction with aspirin in clopidogrel nonresponders.

\section{Methods \\ Study Cohort}

We performed a retrospective cohort study of all patients undergoing treatment of cerebral aneurysms with flow-diverting stents in a tertiary referral center between January 2011 and May 2016. The IRB reviewed and approved the study protocol. All patients received a PED. The characteristics of the cohort at baseline are detailed in Table 1.

\section{Treatment Protocol}

At our institution, patients are pretreated with $75 \mathrm{mg}$ of clopidogrel daily and at least $81 \mathrm{mg}$ of aspirin daily for 10 days before their PED deployment. Some patients presenting for their intervention without having received the appropriate 10 days of DAPT received a loading dose of $325-650 \mathrm{mg}$ of aspirin and a bolus of antiaggregate drug (600 mg of clopidogrel or 40-60 mg of prasugrel or 90 $\mathrm{mg}$ of ticagrelor) less than 24 hours before their intervention. We routinely calculated the P2Y12 platelet inhibition assay (VerifyNow, Accumetrics) for all patients before the procedure. Prasugrel was considered when patients were allergic, nonresponders, or resistant to clopidogrel. Resistance was defined as having $<30 \%$ of platelet P2Y12 receptor inhibition. Twenty-two of the 437 patients did not have a significant platelet inhibition $(<30 \%)$ with clopidogrel, and these constituted our population. Ticagrelor was the final alternative for those whose P2Y12 platelet inhibition was still not satisfactory with prasugrel. After the operation, patients continued to receive $75 \mathrm{mg}$ of clopidogrel daily, $10 \mathrm{mg}$ of prasugrel daily, or $90 \mathrm{mg}$ of ticagrelor
TABLE 1. Patient characteristics

\begin{tabular}{lcc}
\hline \multicolumn{1}{c}{ Characteristic } & Clopidogrel & $\begin{array}{c}\text { Prasugrel/ } \\
\text { Ticagrelor }\end{array}$ \\
\hline No. of patients & 374 & 22 \\
\hline Mean age \pm SD $(\mathrm{yrs})$ & $56.25 \pm 13.29$ & $57.31 \pm 13.55$ \\
\hline Males/females & $357 / 58$ & $16 / 6$ \\
\hline $\begin{array}{l}\text { Mean no. of hypertensive patients } \\
\pm \text { SD }\end{array}$ & $0.51 \pm 0.50$ & $0.54 \pm 0.51$ \\
\hline Mean no. of smokers \pm SD & $0.50 \pm 0.50$ & $0.65 \pm 0.49$ \\
\hline Mean aneurysm size \pm SD (mm) & $9.11 \pm 5.87$ & $12.93 \pm 10.93$ \\
\hline Mean no. w/ previous SAH \pm SD & $0.10 \pm 0.30$ & $0.15 \pm 0.37$ \\
\hline Mean no. w/ adjunctive coiling \pm SD & $0.74 \pm 0.26$ & $0.08 \pm 0.27$ \\
\hline Mean no. of stent migrations \pm SD & $0.01 \pm 0.09$ & $0.04 \pm 0.19$ \\
\hline Mean no. of stents per patient \pm SD & $1.21 \pm 0.56$ & $1.32 \pm 0.57$ \\
\hline $\begin{array}{l}\text { Mean mRS score on last follow-up } \\
\pm \text { SD }\end{array}$ & $0.32 \pm 0.75$ & $0.67 \pm 1.15$ \\
\hline
\end{tabular}

twice daily. Prophylactic antiplatelet therapy was given a minimum of 6 months to 1 year following the procedure.

\section{Outcome Variables}

The key primary outcome was the patients' modified Rankin Scale (mRS) score, which was calculated and registered before the discharge of the patients and at each follow-up visit. Mortality, thromboembolic events, and digital subtraction angiography (DSA)-documented hemorrhagic accidents, following the aneurysm's treatment using a PED, were considered primary outcomes. Secondary outcomes were the postinterventional length of stay and the long-term recurrence.

\section{Patient Follow-Up}

Patients were scheduled for follow-up visits with the senior author at $1,3,6$, and 18 months after their discharge from the institution. The efficacy and safety of the prophylactic DAPT after PED treatment were evaluated on initial postoperative angiography and follow-up angiography when available. Cerebrovascular angiography (DSA) was required at the 6-month visit, and then patients were followed accordingly to evaluate for bleeding recurrence or vessel stenosis. Additional information on the number of PEDs used for initial treatment and on stent migration was collected during follow-up (Table 1). Head CT scans were compared and analyzed by the senior author to document any new or recurrent subarachnoid or intraparenchymal hemorrhage, only if the patients were to develop new insidious symptoms. Medical charts were reviewed to determine whether any retroperitoneal, gastrointestinal, or genitourinary bleeding had occurred.

\section{Exposure Variables}

The primary exposure variable was the treatment (prasugrel or ticagrelor vs clopidogrel). Covariates used for risk adjustment were age and sex. The comorbidities used for risk adjustment were hypertension, diabetes mellitus, smoking, previous subarachnoid hemorrhage (SAH), an- 
eurysm size, periprocedural balloon, and previous coil embolization.

\section{Statistical Analysis}

To investigate the association between clopidogrel exposure and our outcome measures, we used several methods to address confounding. Initially, for binary outcomes we used a multivariable logistic regression controlling for all the covariates mentioned above. To control for clustering at the physician level, we used mixed-effect models with the physician as a random-effects variable. For continuous outcomes, we used the corresponding linear regression analyses.

As an alternate way to control for confounding for binary outcomes, we used a propensity score-adjusted logistic regression model. To derive the propensity of receiving clopidogrel we developed a prediction model using logistic regression, based on all the covariates described above. We subsequently used a logistic regression with adjustment (stratification) by quantiles of the propensity score; we chose the number of quantiles to be 10 . The operating physician was again used as a random-effects variable.

Patients who were lost to follow-up were not included in the original analysis. In sensitivity analysis, all the above analyses were repeated with multiple imputations for the patients lost to follow-up. We created 5 imputed data sets. The directions of the observed associations did not change, and these results are not reported further.

Regression diagnostics were performed for all analyses. Given that the long-term recurrence was $2 \%$ in a study sample of 437 patients, we had an $80 \%$ power to detect a difference in long-term recurrence as small as $13.4 \%$, at an alpha level of 0.05 . All probability values were the result of 2-sided tests. The statistical program Stata (version 13, StataCorp) was used for statistical analysis.

\section{Results}

\section{Demographic Characteristics}

Between 2011 and 2016, a total of 437 patients underwent treatment with PEDs at our institution. The mean age of the patients was 56.3 years, and 62 were women (14.2\%). Twenty-six patients (5.9\%) presented with an acute SAH. Three hundred seventy-four patients received clopidogrel (361 with aspirin, 9 with warfarin, 4 with rivaroxaban), $20(4.6 \%)$ received prasugrel, and 2 received ticagrelor. Seven patients were lost to follow-up after their intervention, 6 from the clopidogrel group and 1 from the prasugrel group. One patient was reported allergic to clopidogrel and prasugrel (Table 1).

\section{Efficacy of Prasugrel and Ticagrelor Intra-PED Stenosis}

Of 374 patients receiving clopidogrel, $23(6.1 \%)$ of the patients had an intra-PED stenosis. In the group of patients receiving prasugrel and ticagrelor, the mean number of intra-PED stenoses was $0.12 \pm 0.33$; only 2 patients had an intra-PED stenosis, $1 / 22(4.5 \%)$ receiving prasugrel, the other ticagrelor. The mean number of intra-PED stenoses in patients receiving clopidogrel was $0.071 \pm 0.26(6.7 \%)$.
TABLE 2. Association between outcomes and clopidogrel

\begin{tabular}{lcc}
\hline \multicolumn{1}{c}{ Variable } & $\begin{array}{c}\text { Multivariable } \\
\text { Regression }\end{array}$ & $\begin{array}{c}\text { Propensity Score } \\
\text { Adjustment }\end{array}$ \\
\hline Long-term recurrence & $0.17(0.01-2.70)$ & $0.26(0.22-3.03)$ \\
\hline OR $(95 \% \mathrm{Cl})$ & 0.21 & 0.28 \\
\hline $\mathrm{p}$ value & & \\
\hline Intra-PED stenosis & $0.44(0.09-2.15)$ & $0.46(0.09-2.44)$ \\
\hline OR $(95 \% \mathrm{Cl})$ & 0.31 & 0.37 \\
\hline $\mathrm{p}$ value & & \\
\hline Thromboembolic complications & $0.46(0.12-1.67)$ & $0.39(0.11-1.41)$ \\
\hline OR $(95 \% \mathrm{Cl})$ & 0.24 & 0.82 \\
\hline $\mathrm{p}$ value & & \\
\hline Hemorrhagic complications & $0.39(0.91-1.64)$ & $0.33(0.08-1.37)$ \\
\hline OR $(95 \% \mathrm{Cl})$ & 0.20 & 0.13 \\
\hline $\mathrm{p}$ value & & \\
\hline Mortality & $2.18(0.11-43.27)$ & $0.73(0.75-7.17)$ \\
\hline OR $(95 \% \mathrm{Cl})$ & 0.61 & 0.79 \\
\hline p value & & \\
\hline
\end{tabular}

A univariate analysis of the effect of clopidogrel on the thromboembolic complication rate did not show any correlation between the 2 variables (OR $0.58,95 \%$ CI $0.12-$ $2.70, p=0.48$ ). In a multivariable mixed-effects logistic regression, clopidogrel was not associated with an increased intra-PED stenosis (OR 0.44, 95\% CI 0.09-2.15, $\mathrm{p}=0.31$ ). This was consistent with the results from the propensity score-adjusted model (OR 0.46, 95\% CI 0.09-2.44, $\mathrm{p}=$ 0.37; Table 2).

\section{Long-Term Recurrence}

None of the 22 patients receiving prasugrel or ticagrelor had a long-term recurrence. Of 374 patients receiving clopidogrel, $1.6 \%$ suffered from a long-term recurrence rate with a mean of $0.02 \pm 0.13$ recurrences. A univariate analysis of the effect of clopidogrel on the long-term recurrence rate did not show any correlation between the 2 variables (OR $0.27,95 \%$ CI $0.03-2.41, p=0.24)$. In a multivariable mixed-effects logistic regression, clopidogrel was not associated with an increased long-term recurrence (OR 0.17, 95\% CI 0.01-2.70, $\mathrm{p}=0.21$ ). This was consistent with the propensity score-adjusted model (OR $0.26,95 \%$ CI 0.22-3.03, $\mathrm{p}=0.28$; Table 2).

\section{Safety of Prasugrel and Ticagrelor \\ Post-PED Complications}

Of 22 patients receiving prasugrel and ticagrelor, the mean number of postprocedural complications was $0.19 \pm$ 0.40 ; only 1 patient developed an arteriovenous $\mathrm{V}_{3}$ fistula, and 1 other patient had an ophthalmoplegia and a ptosis of the left eye. Of 374 patients receiving clopidogrel, the mean number of post-PED complications was $0.53 \pm 0.23$. A univariate analysis of the effect of clopidogrel on the post-PED complication rate was associated with an increased post-PED complication rate (OR $0.24,95 \%$ CI $0.08-0.70, p=0.01$ ). In a multivariable mixed-effects logistic regression, clopidogrel was also associated with an 


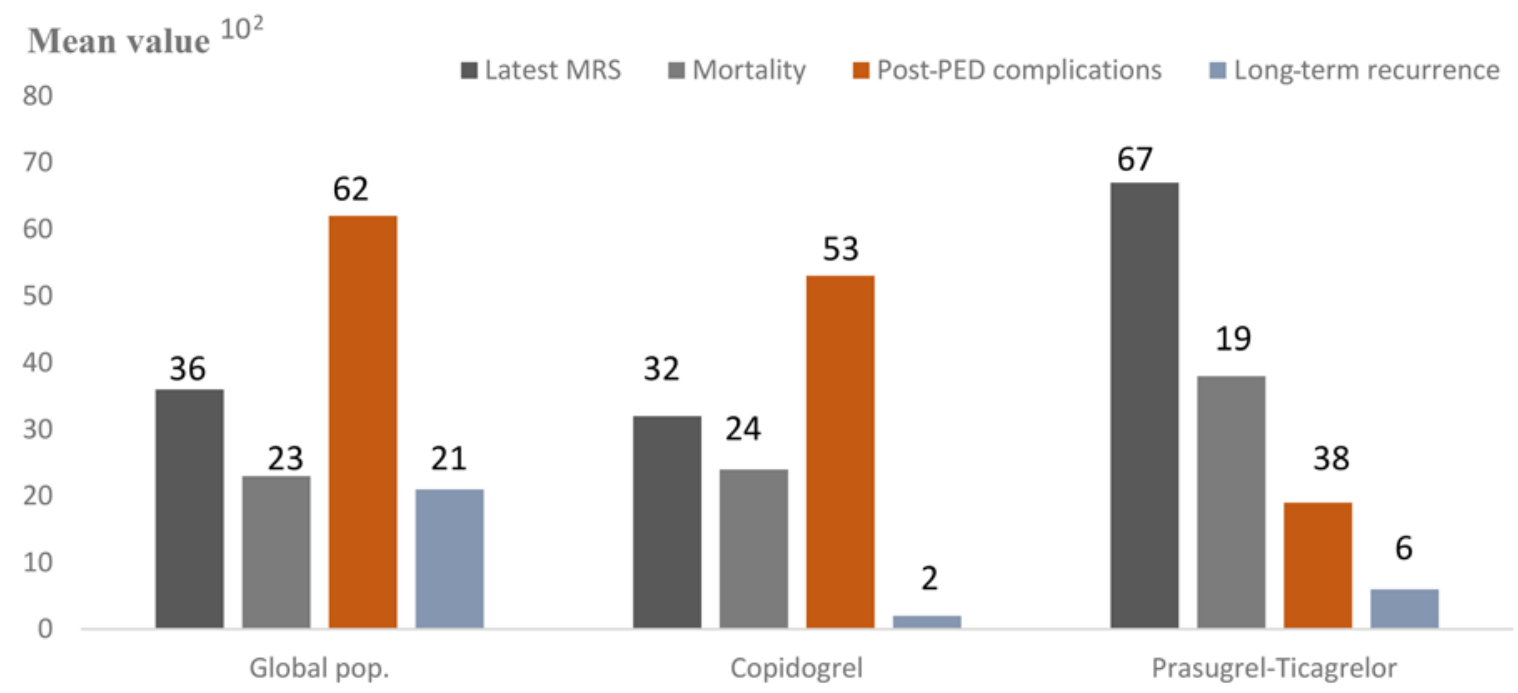

FIG. 1. Graph showing the mean values of the clinical outcomes according to the prescribed antiplatelet drug. pop. = population. Figure is available in color online only.

increased post-PED complication rate (OR $0.28,95 \% \mathrm{CI}$ $0.08-1.01, \mathrm{p}=0.05$ ). This was consistent with the propensity score-adjusted model where the $\mathrm{p}$ value was slightly higher than 0.05 (OR $0.27,95 \%$ CI $0.07-1.03, \mathrm{p}=0.055$ ).

\section{Thromboembolic Complications}

Of 374 patients prescribed clopidogrel, the mean number of thromboembolic events was $0.72 \pm 0.26$. While 28 patients $(7.5 \%)$ receiving clopidogrel had thromboembolic complications, only 1 patient receiving prasugrel experienced word-finding difficulty. A univariate analysis of the effect of clopidogrel on the thromboembolic complication rate did not show any correlation between the 2 variables (OR $0.43,95 \%$ CI $0.14-1.32, \mathrm{p}=0.14$ ). We found similar results in a multivariable mixed-effects logistic regression (OR $0.46,95 \%$ CI $0.12-1.67, \mathrm{p}=0.24$ ) and a propensity score-adjusted model (OR 0.39, 95\% CI 0.11-1.41, p = 0.82 ; Table 2).

\section{Hemorrhagic Complications}

None of the patients receiving prasugrel or ticagrelor suffered from hemorrhagic complications. Of 374 patients receiving clopidogrel, the mean number of hemorrhagic complications was $0.45 \pm 0.21$ (5.6\%). A univariate analysis of the effect of clopidogrel on the hemorrhagic complication rate did not show any correlation between the 2 variables (OR $0.36,95 \%$ CI $0.10-1.33, p=0.13)$. In a multivariable mixed-effect logistic regression, clopidogrel was not associated with an increased hemorrhagic event rate (OR 0.39, 95\% CI 0.91-1.64, $\mathrm{p}=0.20)$. We found similar results with the propensity score-adjusted model (OR $0.33,95 \%$ CI $0.08-1.37, \mathrm{p}=0.13$; Table 2).

\section{Mortality}

Patients receiving clopidogrel had a mean mortality rate of $0.02 \pm 0.15$ (2.67\%; Fig. 1). Nine patients died, including from various non-PED-related events, such as severe sepsis $(\mathrm{n}=1)$, malignant hypertension with large middle cerebral artery infarct $(n=1)$, severe gastrointes- tinal complication $(\mathrm{n}=1)$, and nonreported cause of death $(\mathrm{n}=3)$. Only 3 patients from this group were pronounced dead from acute SAH $(n=2)$ and intraparenchymal hemorrhage $(n=1)$. None of the patients receiving prasugrel and ticagrelor died. A univariate analysis of the effect of clopidogrel on the mortality rate did not show any correlation between the 2 variables (OR $0.61,95 \%$ CI $0.75-5.03$, $\mathrm{p}=0.65$ ). In a multivariable mixed-effect logistic regression, clopidogrel was not associated with an increased mortality rate (OR $2.18,95 \%$ CI $0.11-43.27, \mathrm{p}=0.61)$; this persisted in a propensity score-adjusted model (OR 0.73 , 95\% CI 0.75-7.17, $\mathrm{p}=0.79$; Table 2).

\section{Latest Clinical Status}

Of the 22 patients receiving prasugrel or ticagrelor, all had an mRS score $\leq 2$ on their latest follow-up visit, with a mean score of $0.67 \pm 1.15$. Of the 374 patients receiving clopidogrel, $98.4 \%$ had an mRS score $\leq 2$ at their latest follow-up visit, with a mean score of $0.32 \pm 0.75$ (Fig. 1). In a multivariate analysis in which the latest $\mathrm{mRS}$ score was a dependent variable, clopidogrel did not significantly affect the mRS score on last follow-up ( $p=0.14$, Fig. 1).

\section{Postinterventional Hospital Stay}

Of 22 patients receiving prasugrel or ticagrelor, the mean postinterventional length of stay was $3 \pm 6.20$ days (Fig. 1). Of 374 patients receiving clopidogrel, the mean postprocedural stay was $1.81 \pm 2.67$ days. In a multivariate analysis, clopidogrel did not significantly affect the patients' postoperational length of stay $(\mathrm{p}=0.94)$.

\section{Discussion}

Using a retrospective cohort of candidates with cerebrovascular aneurysms, we did not identify any association between clopidogrel administration and mortality, thromboembolic complications, long-term recurrence, intra-PED stenosis, hemorrhagic events, mRS score on latest followup, and postoperative hospital length of stay. We found 
that clopidogrel is associated with postprocedural complications. Prasugrel and ticagrelor are increasingly used in clopidogrel-resistant individuals treated for their cerebral aneurysms. Compared with clopidogrel, both prasugrel and ticagrelor inhibit platelet aggregation more rapidly and consistently with lower rates of intersubject variability. ${ }^{17}$

In the present study, the efficacy of prasugrel, represented by intra-PED stenosis and long-term recurrence, was roughly similar to that of clopidogrel $(6.1 \%$ vs $4.5 \%$, and $1.6 \%$ vs no recurrence, respectively). These results are consistent with the more favorable pharmacokinetic and pharmacodynamic profiles of prasugrel, which affords a more potent and rapid inhibition of platelet aggregation. ${ }^{1}$ They are also consistent with the TRITON-TIMI 38 (Trial to Assess Improvement in Therapeutic Outcomes by Optimizing Platelet Inhibition with Prasugrel-Thrombolysis In Myocardial Infarction 38) study, in which clopidogrelnaïve patients with acute coronary syndrome scheduled for percutaneous coronary intervention on prasugrel therapy showed significantly reduced rates of ischemic events, including patients with cardiac stent thrombosis..$^{18}$ A recent meta-analysis performed by Patti et al. found that in patients undergoing percutaneous coronary intervention, switching from clopidogrel to prasugrel tended to decrease the incidence of major adverse cardiac events during follow-up. ${ }^{11}$ Despite the lack of clear evidence supporting its use in cerebrovascular procedures, Leslie-Mazwi et al. were the first to report the successful use of prasugrel for acute in-stent thrombosis in a patient with reduced clopidogrel response undergoing elective stent-assisted aneurysm coil embolization. ${ }^{8}$

In our series, patients receiving aspirin and prasugrel did not have any hemorrhagic complications. The small number of patients who were receiving both aspirin and prasugrel could explain these results, as the use of DAPT with aspirin and prasugrel would be expected to increase the relative risk of bleeding by $30 \%$ compared with aspirin and clopidogrel. ${ }^{918}$ Interestingly, the greatest benefit with prasugrel compared with clopidogrel in the TRITONTIMI 38 study was noted in high-risk patients, especially patients with diabetes or those who suffered an ST-segment elevation myocardial infarction, in which the reduction in major adverse cardiac events with prasugrel was not paralleled by an increased risk of bleeding. ${ }^{11}$ This may imply that there are certain subgroups of patients who are at a decreased risk of hemorrhagic adverse events from prasugrel use. The incidence of thromboembolic complications was approximately similar in the aspirin/clopidogrel group (7.4\%) and in the aspirin/prasugrel group (5\%). It was not similar to the extrapolated results of many studies present in the cardiac literature that demonstrated superior reduction of ischemic events using prasugrel as part of DAPT compared with clopidogrel.., 18

The ticagrelor/aspirin combination was used only in 2 patients who either did not achieve the desired P2Y12 platelet inhibition with prasugrel or were allergic to it. One patient had an intra-PED stenosis and another suffered from a postprocedural hemorrhagic complication manifesting as mild aphasia. Conclusions about the efficacy and safety of ticagrelor in patients with PEDs cannot be drawn from our series because of our limited num- ber of patients. In their series of 18 patients, Hanel et al. presented their successful experience with patients using ticagrelor for different neuroendovascular procedures as an alternative to clopidogrel in nonresponders. ${ }^{6}$ Further investigations in patients undergoing treatment with PEDs and other neuroendovascular procedures are needed to assess the efficacy and safety profile of ticagrelor in hyporesponders and nonresponders to clopidogrel.

It is noteworthy to state that our patients followed up over a mean of $15.8 \pm 12.4$ months have shown no regression but an increasing improvement of the patients' clinical well-being. All our patients had an mRS score $\leq 2$ and their mean length of stay in the hospital was approximately 3 days, with $65 \%$ discharged within 2 days. These data are consistent with those from the series of Stetler et al., who also was able to discharge patients on prasugrel on postoperative Day $1 .{ }^{15} \mathrm{We}$ believe that prasugrel would be not only efficacious, but also safe when dispensed in this context. This patient-safety model is definitely multifactorial and it might not be clearly related to the use of prasugrel. However, we might infer that prasugrel would not be adversely interfering with the patient's clinical well-being.

There is still no clear indication for the use of prasugrel as an alternative treatment for patient resistance to clopidogrel during the placement of a PED. The main concern of clinicians is the increased bleeding risk associated with its use, as shown in several cardiovascular studies. ${ }^{11,18}$ However, the difference in end-organ result response (brain vs cardiac muscle), tortuosity of intracranial vasculature, and the amount of metal implanted make it ineffective to simply apply cardiac literature to intracranial procedures. ${ }^{6}$ Akbari et al. ${ }^{1}$ presented their experience with prasugrel and aspirin in a cohort of 25 patients undergoing different neuroendovascular procedures, 9 of whom underwent PED placement. These investigators observed a significant increase in hemorrhagic complications $(19.4 \%$ vs $3.6 \%$, p $=0.02$ ) in the prasugrel/aspirin group as compared with patients treated with clopidogrel/aspirin. Jones et al. tried using low-dose prasugrel in 2 cases following PED implantation in patients who showed hyporesponsiveness to clopidogrel. Both patients did well with no thromboembolic or hemorrhagic complications. ${ }^{7}$ Our series of patients treated with PED placement who were started on prasugrel due to hyporesponsiveness to clopidogrel is the largest so far. We did not observe any ischemic events related to thromboembolism or in-stent thrombosis. We also did not find an increased risk of bleeding in those patients. Whether our patients fall into a subgroup of patients that have a lower propensity to experience bleeding complications with prasugrel or these results are due to chance alone is not clear. Conclusions cannot be drawn at this level, and more investigations should be warranted to study the efficacy and safety of prasugrel in patients treated endovascularly with PED placement who are hyporesponders to clopidogrel. The higher cost of prasugrel than of clopidogrel should also be taken into consideration when prescribing the drug. ${ }^{5}$

\section{Limitations of the Study}

While our series is one of the largest to date documenting the safety and efficacy of prasugrel in the endovascular 
PED setting, our study design is limited by the small sample size and by the retrospective nature of data collection. None of the patients receiving prasugrel manifested major adverse events. This does not definitively show that prasugrel is as effective as clopidogrel in the PED patient population, and our results could not be extrapolated to all the neurointervention specialized centers. Further randomized clinical trials are indispensable to investigate the promising outcome of these drugs to determine if they could replace clopidogrel in patients receiving PED flow-diversion treatment.

\section{Conclusions}

Patients undergoing PED treatment require optimal platelet inhibition. The key to ensuring these patients' long-term clinical well-being is applying the right P2Y12 platelet inhibitor before and after PED deployment. Approximately $30 \%$ of the patients receiving clopidogrel are heterozygous for the $C Y P 2 C 19$ gene and show a hyporesponsiveness or resistance $(<30 \%$ platelet inhibition). We administered prasugrel and ticagrelor in this series of clopidogrel-resistant patients and endorsed the reasonable long-term clinical well-being of our patients. Prasugrel should be an adequate substitute in clopidogrel-resistant and -allergic patients treated with PEDs for their intracranial aneurysms.

\section{References}

1. Akbari SH, Reynolds MR, Kadkhodayan Y, Cross DT III, Moran CJ: Hemorrhagic complications after prasugrel (Effient) therapy for vascular neurointerventional procedures. J Neurointerv Surg 5:337-343, 2013

2. Delgado Almandoz JE, Kadkhodayan Y, Crandall BM, Scholz JM, Fease JL, Tubman DE: Variability in initial response to standard clopidogrel therapy, delayed conversion to clopidogrel hyper-response, and associated thromboembolic and hemorrhagic complications in patients undergoing endovascular treatment of unruptured cerebral aneurysms. J Neurointerv Surg 6:767-773, 2014

3. Dobesh PP, Varnado S, Doyle M: Antiplatelet agents in cardiology: a report on aspirin, clopidogrel, prasugrel, and ticagrelor. Curr Pharm Des 22:1918-1932, 2016

4. Guédon A, Clarençon F, Di Maria F, Rosso C, Biondi A, Gabrieli J, et al: Very late ischemic complications in flow-diverter stents: a retrospective analysis of a single-center series. J Neurosurg 125:929-935, 2016

5. Gupta R, Moore JM, Griessenauer CJ, Adeeb N, Patel AS, Youn R, et al: Assessment of dual-antiplatelet regimen for Pipeline Embolization Device placement: a survey of major academic neurovascular centers in the United States. World Neurosurg 96:285-292, 2016

6. Hanel RA, Taussky P, Dixon T, Miller DA, Sapin M, Nordeen JD, et al: Safety and efficacy of ticagrelor for neuroendovascular procedures. A single center initial experience. J Neurointerv Surg 6:320-322, 2014

7. Jones GM, Twilla JD, Hoit DA, Arthur AS: Prevention of stent thrombosis with reduced dose of prasugrel in two patients undergoing treatment of cerebral aneurysms with pipeline embolisation devices. J Neurointerv Surg 5:e38, 2013

8. Leslie-Mazwi TM, Chandra RV, Oh DC, Nogueira RG: Novel use of prasugrel for intracranial stent thrombosis. J Neurointerv Surg 3:358-360, 2011

9. Mega JL, Close SL, Wiviott SD, Shen L, Hockett RD, Brandt
JT, et al: Cytochrome P450 genetic polymorphisms and the response to prasugrel: relationship to pharmacokinetic, pharmacodynamic, and clinical outcomes. Circulation 119:2553-2560, 2009

10. Molyneux A, Kerr R, Stratton I, Sandercock P, Clarke M, Shrimpton J, et al: International Subarachnoid Aneurysm Trial (ISAT) of neurosurgical clipping versus endovascular coiling in 2143 patients with ruptured intracranial aneurysms: a randomised trial. Lancet 360:1267-1274, 2002

11. Patti G, Ricottini E, De Luca L, Cavallari I: Safety and efficacy of switching from clopidogrel to prasugrel in patients undergoing percutaneous coronary intervention: a study-level meta-analysis from 15 studies. J Cardiovasc Pharmacol 67:336-343, 2016

12. Pistocchi S, Blanc R, Bartolini B, Piotin M: Flow diverters at and beyond the level of the circle of Willis for the treatment of intracranial aneurysms. Stroke 43:1032-1038, 2012

13. Qureshi AI, Luft AR, Sharma M, Guterman LR, Hopkins LN: Prevention and treatment of thromboembolic and ischemic complications associated with endovascular procedures: Part I-Pathophysiological and pharmacological features. Neurosurgery 46:1344-1359, 2000

14. Qureshi AI, Luft AR, Sharma M, Guterman LR, Hopkins LN: Prevention and treatment of thromboembolic and ischemic complications associated with endovascular procedures: Part II-Clinical aspects and recommendations. Neurosurgery 46:1360-1376, 2000

15. Stetler WR, Chaudhary N, Thompson BG, Gemmete JJ, Maher CO, Pandey AS: Prasugrel is effective and safe for neurointerventional procedures. J Neurointerv Surg 5:332-336, 2013

16. Tan LA, Keigher KM, Munich SA, Moftakhar R, Lopes DK: Thromboembolic complications with Pipeline Embolization Device placement: impact of procedure time, number of stents and pre-procedure P2Y12 reaction unit (PRU) value. J Neurointerv Surg 7:217-221, 2015

17. Weerakkody GJ, Jakubowski JA, Brandt JT, Farid NA, Payne $\mathrm{CD}$, Zhu J, et al: Comparison of speed of onset of platelet inhibition after loading doses of clopidogrel versus prasugrel in healthy volunteers and correlation with responder status. Am J Cardiol 100:331-336, 2007

18. Wiviott SD, Braunwald E, McCabe CH, Montalescot G, Ruzyllo W, Gottlieb S, et al: Prasugrel versus clopidogrel in patients with acute coronary syndromes. N Engl J Med 357:2001-2015, 2007

\section{Disclosures}

Drs. Tjoumakaris and Jabbour report being consultants for Medtronic.

\section{Author Contributions}

Conception and design: Jabbour, Atallah, Chalouhi. Acquisition of data: Atallah. Analysis and interpretation of data: Atallah, Saad. Drafting the article: Atallah. Critically revising the article: Jabbour, Atallah, Chalouhi. Reviewed submitted version of manuscript: Jabbour, Hasan, Eller, Stidd. Approved the final version of the manuscript on behalf of all authors: Jabbour. Statistical analysis: Bekelis. Administrative/technical/material support: Atallah, Saad, Tjoumakaris, Hasan, Eller, Stidd, Rosenwasser. Study supervision: Jabbour, Chalouhi, Tjoumakaris, Rosenwasser.

\section{Correspondence}

Pascal Jabbour, Department of Neurological Surgery, Thomas Jefferson University Hospital, 901 Walnut St., 3rd Fl., Philadelphia, PA 19107. email: pascal.jabbour@jefferson.edu. 